\title{
Impacts of grassland fence on the behavior and habitat area of the critically endangered Przewalski's gazelle around the Qinghai Lake
}

\author{
YOU ZhangQiang ${ }^{1,2,3}$, JIANG ZhiGang ${ }^{1 *}$, LI ChunWang ${ }^{1} \&$ MALLON David $^{4}$ \\ ${ }^{1}$ Key Laboratory of Animal Ecology and Conservation Biology, Institute of Zoology, Chinese Academy of Sciences, Beijing 100101, China; \\ ${ }^{2}$ University of Chinese Academy of Sciences, Beijing 100049, China; \\ ${ }^{3}$ Mianyang Normal University, Mianyang 621000, China; \\ ${ }^{4}$ Antelope Specialist Group, SSC, IUCN/Dept of Biology, Chemistry and Health Science, Manchester Metropolitan University, \\ Manchester M1 5GD, UK
}

Received November 9, 2012; accepted February 25, 2013; published online May 22, 2013

\begin{abstract}
The trend of fencing grassland as livestock paddocks is spreading on the Eurasian steppe, however, its impacts on grassland wildlife are little known. In order to explore such impacts, we carried out a field study on how grassland fencing impacts Przewalski's gazelle (Procapra przewalskii), a species listed as EN (Endangered) by SSC/IUCN, on the Qinghai-Tibet Plateau. The results revealed that (1) in the fenced areas, daily movement distance of Przewalski's gazelle was $5081 \pm 1187 \mathrm{~m}$ (Hudong-Ketu) and $4110 \pm 912 \mathrm{~m}$ (Yuanzhe), which was much shorter than the $7223 \pm 546 \mathrm{~m}$ recorded in an unfenced area (Kuaierma); (2) the feeding bout duration of Przewalski's gazelle was much shorter in the fenced habitat; (3) the frequency of walking along both high or low fence lines reached about $81 \%$; while the frequency of jumping across the low fence line was only about $1.2 \%$ and frequency of crawling through the bottom of the high fence lines was about $17.8 \%$; (4) the size of post-fencing habitat decreased to about $20 \%$ and $6 \%$ of the sizes of pre-fencing habitat in Hudong-Ketu and Yuanzhe areas respectively, but no clear change in the size of habitat area was found in the unfenced Kuaierma area; and (5) the fence lines impaired the possibility of gazelles to escape from predators and occasionally trapped the Przewalski's gazelle which failed to jump over the fence lines. Death occurrence of Przewalski's gazelle in the intensively fenced area, including gazelles strangled by fence lines and predated by wolves, reached $5 \%$ of the population size in Yuanzhe and up to 15\%-20\% in Hudong-Ketu. This study highlights the negative impacts of grassland fencing on Przewalski's gazelle and proposes measures for integrating conservation of this gazelle with livestock management practice.
\end{abstract}

Procapra przewalskii, fence line, habitat, livestock, range management

Citation: You Z Q, Jiang Z G, Li C W, et al. Impacts of grassland fence on the behavior and habitat area of the critically endangered Przewalski's gazelle around the Qinghai Lake. Chin Sci Bull, 2013, 58: 2262-2268, doi: 10.1007/s11434-013-5844-9

Linear infrastructures such as railroads, highways, pipelines and fence lines have significant effects on wildlife [1-3]. These include degradation and reduction of habitat, restricted access to forage or other key resources, blocked migration routes, improved access for poachers, genetic isolation, and direct death occurrence from trying to cross [4].

In order to increase livestock productivity, herders are now turning grasslands into fenced paddocks. During our field expeditions in other areas in China since the mid-

*Corresponding author (email: jiangzg@ioz.ac.cn) 1990s, we have found grassland fencing is spreading widely in the country, from Manzhouli in northeast China to Xinjiang in the northwest and to the Qinghai-Tibet Plateau, an indication of intensive management of the once pastoral ranges. Now, as part of agricultural modernization, more and more livestock paddocks were built in Chinese grassland. Up to 2002, about $70 \%$ of China's grasslands [6,7], which account for $18 \%$ of the country's total territory were fenced. Many studies had suggested that the grassland fence lines have disadvantage of restricting animal movements, access to forage and other resources of wild ungulate spe- 
cies $[5,8,9]$. However, the ecological consequences of such a large scale environmental modification are unknown.

Przewalski's gazelle used to be found in Gansu, Inner Mongolia, Ningxia and Qinghai in western China [13-16]. However, due to habitat fragmentation and loss, it is now confined to areas around the Qinghai Lake basin on the north-east edge of the Qinghai-Tibet Plateau, and except for about 100 Przewalski's gazelles lately discovered in Kuaierma, Tianjun County [17], fewer than 300 individuals of Przewalski's gazelle remained free ranging in 1999 $[15,18]$. Liu and Jiang [19] reported that almost all habitats of this gazelle had been fenced as livestock paddocks in Hudong-Ketu and Yuanzhe. It was suggested that grassland fencing had negative impacts on Przewalski's gazelle [19-21]. Recently, more populations of the Przewalski's gazelle were discovered; however, those populations were engulfed by pasture fence lines [22]. You et al. [23] reported pasture paddock fence affected the shape and size of breeding territories of the Przewalski's gazelle.

The primary aim of this study was to assess the impacts of grassland fencing on activity patterns, daily movement distances and habitat loss of Przewalski's gazelle. We also recorded the number of gazelle death caused by grassland fence lines and wolf predation near fence lines. Finally, we provide recommendations concerning establishment of a nature reserve for the conservation of the endangered Przewalski's gazelle, modifications to the design of grassland fences and the need to integrate wild animal conservation into grassland livestock management.

\section{Methods}

\subsection{Study areas}

Field work was carried out at three sites: Hudong-Ketu, Yuanzhe and Kuaierma, around Qinghai Lake (38 $25^{\prime}-$ $36^{\circ} 28^{\prime} \mathrm{N}, 97^{\circ} 53^{\prime}-101^{\circ} 13^{\prime} \mathrm{E}$, Figure 1). The landscape of Hudong-Ketu is a mosaic of steppe, alpine shrub, alpine meadow and sand dunes whereas that of Yuanzhe and Kuaierma is composed of steppe and alpine meadow. The steppe, alpine shrub and alpine meadows in Hudong-Ketu and Yuanzhe have been fenced with wire netting since the early 1990s [14]. There is no fenced grassland in Kuaierma which is located in the remote upper reaches of the Buha River, and we chose this site as a control area. Pastoralism is still practiced in the study areas; Tibetan and Mongolian herdsmen move their livestock to mountain slopes in summer and return with the livestock to the valleys and the lake shore at lower altitudes in winter. We carried out field investigation in Hudong-Ketu, Yuanzhe and Kuaierma from

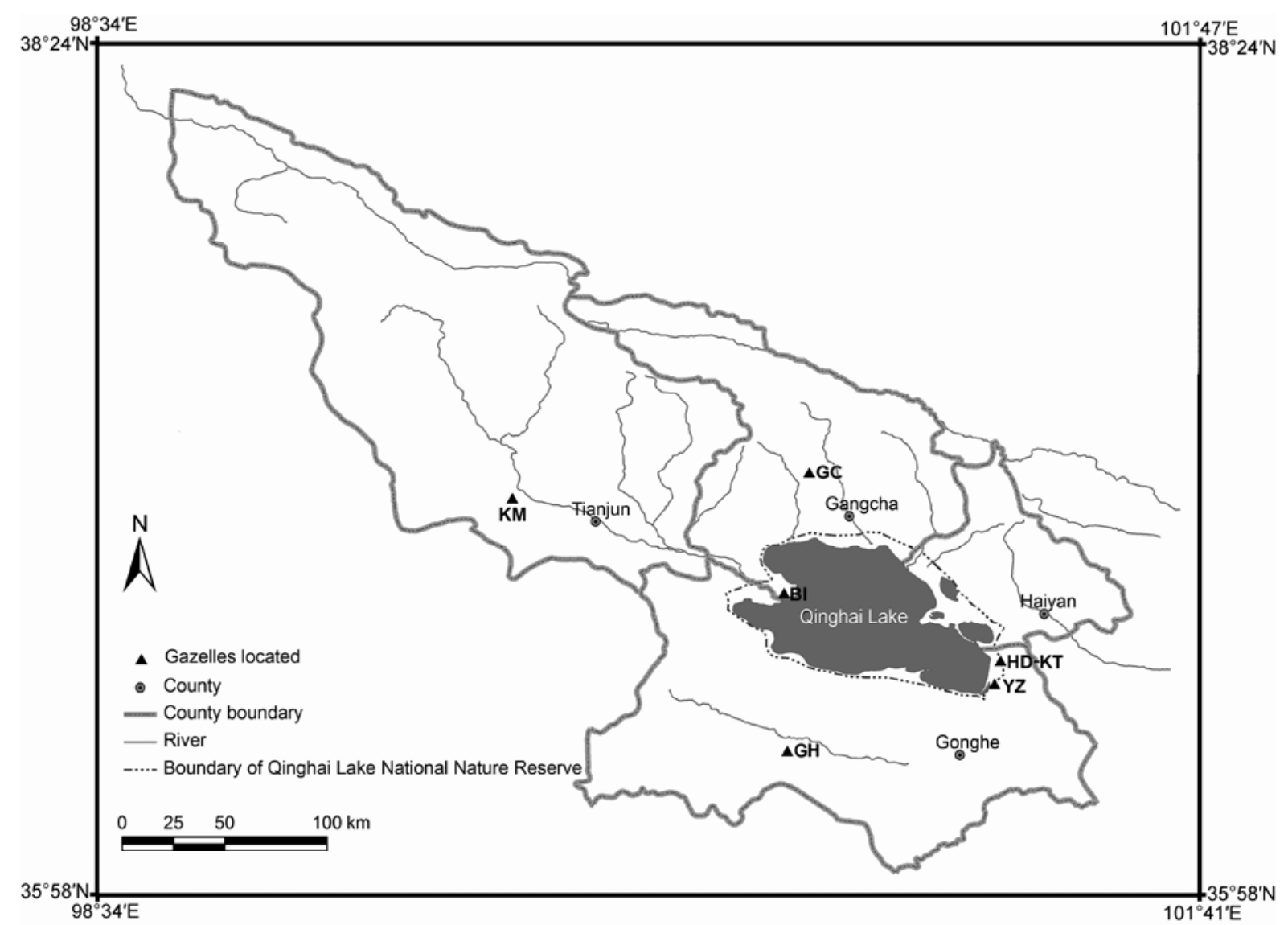

Figure 1 Current distribution status of the Przewalski's gazelle around Qinghai Lake in northwest China. There are six populations: Gonghe (GH), Gangcha (GC), Bird Island (BI), Hudong-Ketu (HD-KT), Yuanzhe (YZ) and Kuaierma (KM), among which the Hudong-Ketu, Yuanzhe and Kuaierma population were studied in this paper. 
2002 to 2005 .

\subsection{Study populations}

There are now six isolated small populations of Przewalski's gazelle surviving in Hudong-Ketu, Yuanzhe, Gangcha, Kuaierma, Bird Island and Shadao in the Qinghai Lake basin (Figure 1). We studied the Hudong-Ketu, Yuanzhe and Kuaierma populations, as these contained $60 \%$ of all Przewalski's gazelles. At the beginning of the study, about 90 gazelles moved between sand dunes and other types of fenced habitat to graze in Hudong-Ketu. Habitat of the Yuanzhe population had been completely fenced and the population size was about 30 . We did not study the Przewalski's gazelle in the unfenced grassland at Kuaierma until 2004; we investigated the range and density of Przewalski's gazelle in the area before 2004 by interviewing local Tibetan pastoralists. The gazelle population size was about 120 in Kuaierma in 2004.

\subsection{Grassland fence lines, daily movement distance and habitat area}

Grassland fencing was designed in two categories, low grassland fencing (1.2 $\mathrm{m}$ high in design) and high grassland fencing (1.5 m high in design). However, height of all the grassland fencing varied after being used for some years. In order to measure the actual mean height of grassland fencing, we sampled 20 and 14 fence lines in Hudong-Ketu and Yuanzhe respectively. We measured vertical height of each sampled grassland fence at three different positions at an interval of $100 \mathrm{~m}$ and treated the mean of the three heights as the height of the corresponding fence line.

To measure the density of grassland fence lines, we firstly recorded the geographic coordinates of each fence line with a GPS (Garmin GPS $72 \mathrm{H}$ ) receiver during the field investigation. All the geographic coordinates were plotted on a digitized map of the study areas using ArcView $3.2^{\circledR}$ to create linear features (Figure 2). Secondly, we measured the minimum distance (vertical distance between two adjacent fence lines) between each two fence lines of the two study areas using GIS tools. In total, distances between 24 and 11 adjacent fence lines were treated in $\mathrm{Hu}$ dong-Ketu and Yuanzhe respectively. Finally we used mean distances between the two adjacent fence lines as grassland fence line densities.

Telemetry was not permitted on this endangered species; therefore between 2002 and 2005 we monitored daily movement distances by tracking and recorded the location of individual and/or flock tracks with GPS. A flock was defined as a number of individuals observed in substantially closer proximity to one another than to other members of the population, and potentially able to communicate and interact cohesively with, and respond to the actions of other members of the flock. Shape of horn and speckle on throat were used to identify sexual matured male single flock. We did not monitor daily movement distances of single female and lamb because single flock of them was seldom observed in field. Population size of the gazelle between 2002 and 2005 were monitored by using the same method mentioned above. To measure the daily movement distance, we marked the site where we found the individual and/or flock at dawn and then tracked their movements until dusk. In order to avoid disturbing the gazelles, we maintained a distance of about $200 \mathrm{~m}$ away from them during the whole tracking time. We measured 30 and 25 tracks of Przewalski's gazelles in Hudong-Ketu and Yuanzhe, respectively. Ten track lines of daily movement distance were measured in Kuaierma. All geographical coordinates of those track lines were plotted on the digitized map to create linear features using ArcView $3.2^{\circledR}$ as described above. We measured daily movement distance of Przewalski's gazelles on the map. Mean length of those linear features was treated as the daily movement distance.

Current habitat area of Przewalski's gazelle was defined as anywhere we found individuals or any tracks of the gazelles. We set out two line transects to investigate habitat area and population size of Przewalski's gazelle in each study area. One line transect was set within the transitional zone of grassland and sand dunes and another in the fenced grassland in Hudong-Ketu. Both transects were set within grassland in Yuanzhe (fenced) and Kuaierma (unfenced). Line transects were surveyed on foot at approximately 1000 $\mathrm{m} / \mathrm{h}$ at 11:00-13:00 and 17:00-19:00 when the gazelles were active and we marked tracks of Przewalski's gazelles using GPS (Garmin GPS72 H). We put all the geographic coordinates of activity tracks of the gazelle on a digitalized map of study area and using ArcView $3.2^{\circledR}$ to create polygon features for measuring current habitat area of the gazelle and the largest size one was used as the habitat area in the all three study sites.

In order to test the changes of habitat area of the gazelle in Hudong-Ketu and Yuanzhe, we compared historical habitat area of this species described by Prejevalsky (Przewalsky) [24], Jiang et al. [14], and Li and Jiang [25]. We also checked the population monitoring data obtained by our group since 1994.

\subsection{Feeding bout, and behavioral responses to grass- land fences}

A feeding bout was defined as when continuous grazing was broken for at least three minutes. We recorded feeding bouts of gazelles in the fenced and unfenced areas in summer and winter 2005. Behaviors of focal gazelles were continuously recorded during $10 \mathrm{~min}$ observation period; followed by 10 min break before the next bout of observation $[21,23]$. Total field behavioral observation times were 354 and $368 \mathrm{~h}$ in the fenced habitat (Hudong-Ketu and Yuanzhe) and unfenced habitat (Kuaierma), respectively.

Behavioral sampling was conducted in Hudong-Ketu ar- 
ea in summer and winter of 2002 and in Yuanzhe in winter of 2003, and in summer and winter of 2004 at Kuaierma. During the scans, we recorded gazelles jumping across fences (JC), crawling through fences (CT) and walking along fences (WA) at each ten minute period during day time. Activity of focal gazelles recorded during a ten minute scan period; followed by a ten minute break before next bout of scan. JC was defined as when a gazelle leapt from one side of a fence to the other; CT was defined as when a gazelle crawled through a place where the fence line was loosely attached to poles; WA was defined as a gazelle walked along a fence line.

\subsection{Death occurrence}

Death occurrence of Przewalski's gazelles on or near the grassland fence was recorded during the field work. Occasionally, gazelles were trapped by the grassland fences when they attempted to jump across the fence line. Such death occurrence was recorded year-round. Death occurrence due to wolf predation occurred mainly in winter during the rutting season of the gazelle [21], when wolves followed livestock back to their winter pastures where they overlapped with the habitat of Przewalski's gazelle. We ascertained that remains of gazelles killed at sites near fences were predated by wolves according to the presence of foot prints of wolf and bites on the carcass.

\subsection{Data analysis}

Because the distribution of data differed significantly from a normal distribution (one sample Kolmogorov-Smirnov test, $P<0.05)$, and the data were still not normally distributed after transformation; we used Mann-Whitney $U$-test to check differences in activity patterns of Przewalski's gazelle in relation to high and low fences. Differences in feeding bout duration and daily movement distance in fenced and unfenced habitat were also tested using the Mann-Whitney $U$-test. Correlation between behavioral responses of Przewalski's gazelle and height of grassland fence was tested by Spearman correlation procedure. In this correlation analysis, there were 34 samples of fence height we measured in Hudong-Ketu and Yuanzhe respectively. We also used Spearman correlation to analyze correlation between daily movement distance and grassland fence density. We pooled the behavioral data of gazelles from the fenced grasslands of Hudong-Ketu and Yuanzhe to analyze differences in behavioral response to grassland fencing. A difference at $P<0.05$ was taken as significant for all statistical tests and all analyses were carried out with SPSS 13.0.

\section{Results}

\subsection{Grassland fence}

All steppe, alpine shrub and alpine meadow habitats are fenced in Hudong-Ketu and Yuanzhe areas, whereas no paddock fences have been built in the Kuaierma area (Figure 2). Due to the top and bottom wires sometimes being loosely attached to the poles, the actual height of grassland fence decreased compared with their height in design in fenced areas (Table 1).
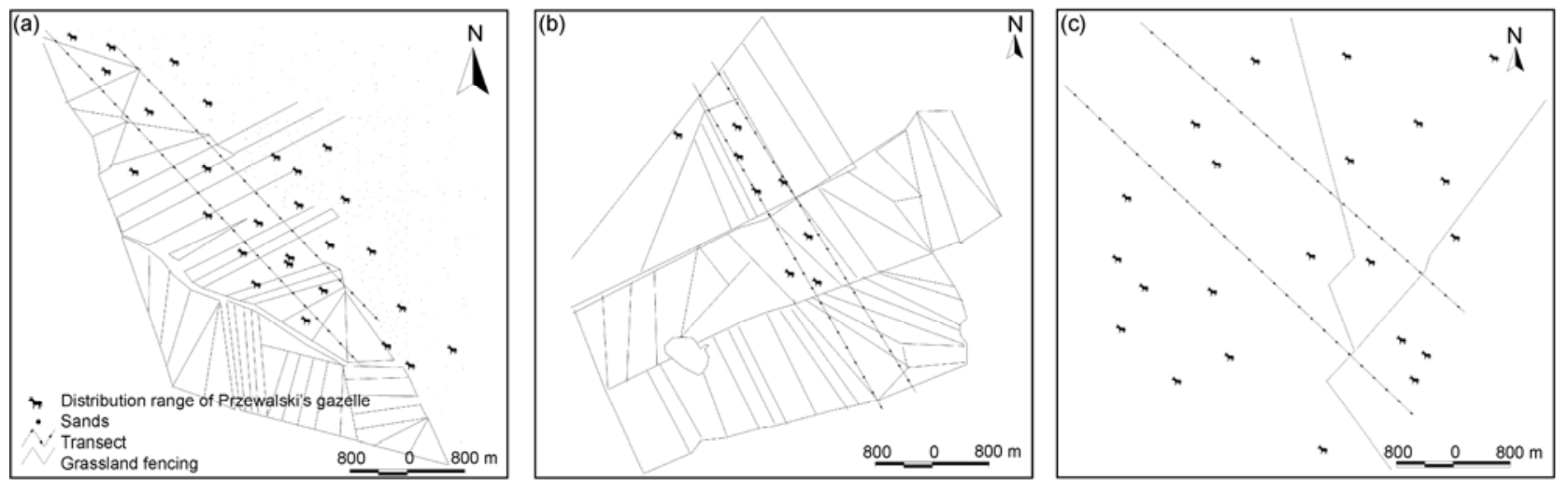

Figure 2 Fenced grassland paddocks and habitat area of Przewalski's gazelle in the three study areas around the Qinghai Lake region. (a) Hudong-Ketu area; (b) Yuanzhe area; (c) Kuaierma area. The arrowed lines indicate the survey transects in the study areas.

Table 1 Description of study areas

\begin{tabular}{lccccccc}
\hline & $\begin{array}{c}\text { Fence height }(\mathrm{m}) \\
(\mathrm{Mean} \pm \mathrm{SE})\end{array}$ & $\begin{array}{c}\text { Fence density } \\
(\text { fence lines/km) } \\
(\mathrm{Mean} \pm \mathrm{SE})\end{array}$ & $\begin{array}{c}\text { Date of } \\
\text { fencing }\end{array}$ & $\begin{array}{c}\text { Habitat area before fenced } \\
\text { for livestock }\left(\mathrm{hm}^{2}\right)\end{array}$ & $\begin{array}{c}\text { Current habitat } \\
\text { area }\left(\mathrm{hm}^{2}\right)\end{array}$ & $\begin{array}{c}\text { Gazelle } \\
\text { population size } \\
(\text { Mean } \pm \mathrm{SE})\end{array}$ & $\begin{array}{c}\text { Density } \\
\left(\mathrm{hm} \mathrm{m}^{2} \text { per gazelle) }\right.\end{array}$ \\
\hline Hudong-Ketu & $1.01 \pm 0.09$ & $30.9 \pm 16.0$ & 1995 & 75956.00 & 1591.20 & $93 \pm 12$ & 17.10 \\
Yuanzhe & $1.06 \pm 0.09$ & $32.7 \pm 7.0$ & 1995 & 7963.33 & 477.80 & $34 \pm 7$ & 14.05 \\
Kuaierma & \multicolumn{2}{c}{ Non-fenced } & & 18955.00 & $120 \pm 23$ & 157.95 \\
\hline
\end{tabular}




\subsection{Current habitat area}

The current habitat area of gazelles in fenced habitat was much smaller than that in the unfenced habitat. The pergazelle habitat area of unfenced habitat (Kuaierma) was about $157.95 \mathrm{hm}^{2}$, which was about ten times that in fenced habitat (Hudong-Ketu and Yuanzhe) (Table 1 and Figure 3).

\subsection{Daily movement distance}

Daily movement distances of the gazelle in the unfenced area (Kuaierma) were longer than those in fenced area $\mathrm{CHu}-$ dong-Ketu and Yuanzhe) and there are significant variance in daily movement distance between fenced habitat and unfenced habitat in Przewalski's gazelle (Mann-Whitney Test, $U=5.27, d f=63, P=0.037$ ) (Table 2).

\subsection{Behavioral responses to grassland fences}

Feeding bout duration of Przewalski's gazelles in fenced habitat (both Hudong-Ketu and Yuanzhe) was significantly shorter than in unfenced habitat (Kuaierma) (Table 2). JC and CT differed significantly in relation to fences height
(Mann-Whitney Test for JC, $U=35.280, r=-0.800, P=0.041$; Mann-Whitney Test for CT, $U=45.00, r=-0.710, P=0.035)$; however, WA did not vary with respect to fence height (Mann-Whitney Test for WA, $U=20.400, r=0.070, P=0.510$ ) (Table 3).

\subsection{Death occurrence caused by fencing and wolf predation}

Three Przewalski's gazelles were found died on grassland fence direclty duirng the study period. Two of them were males and another one was a pregnant female. We found 15 remains of Przewalski's gazelle killed by wolves in the ecotone of the fenced steppe and sand dunes in HudongKetu. Death occurrence of Przewalski's gazelle in the intensively fenced area reached 5\% in Yuanzhe and up to $15 \%-$ $20 \%$ in Hudong-Ketu.

\section{Discussion}

Our results suggest that grassland fencing had clear impact on Przewalski's gazelle. Firstly, habitat area of gazelles in

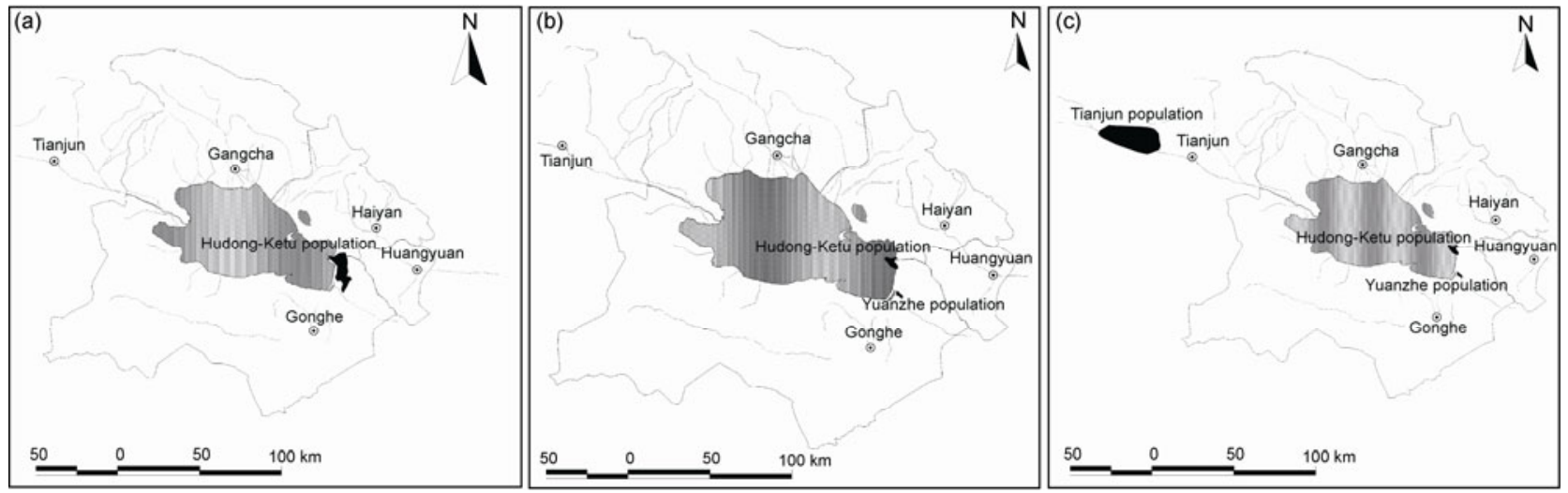

Figure 3 Change of habitat areas of Przewalski's gazelle from 1994 to 2005. (a) Habitat area of Przewalski's gazelle in Hudong-Ketu area in 1994; (b) habitat area of Przewalski's gazelle in Hudong-Ketu and Yuanzhe areas in 2002; (c) current habitat area of Przewalski's gazelle in Hudong-Ketu, Yuanzhe and Kuaierma areas.

Table 2 Impact of grassland fence on Przewalski's gazelle ${ }^{\text {a) }}$

\begin{tabular}{|c|c|c|c|c|c|c|c|c|c|c|}
\hline & \multirow{2}{*}{ Study area } & \multirow{2}{*}{$\begin{array}{l}\text { Fence type } \\
\text { in design }\end{array}$} & \multirow{2}{*}{ Death } & \multirow{2}{*}{$\begin{array}{l}\text { Daily movement distance (m) } \\
\quad(\text { Mean } \pm \mathrm{SE}, n)\end{array}$} & \multicolumn{3}{|c|}{ Remains of gazelle } & \multirow{2}{*}{ Casualty } & \multirow{2}{*}{$\begin{array}{c}\text { Death } \\
\text { occurrence }\end{array}$} & \multirow{2}{*}{$\begin{array}{l}\text { Feeding bout } \\
\text { length }(\mathrm{n} / \mathrm{m}) \\
(\mathrm{Mean} \pm \mathrm{SE})\end{array}$} \\
\hline & & & & & 1 st rut & 2nd rut & 3 rd rut & & & \\
\hline \multirow[t]{4}{*}{ Fenced area } & Hudong-Ketu & high fence & + & $5081 \pm 1187(30)$ & 4 & 6 & 5 & 1 & $15 \%-20 \%$ & $36 \pm 8$ \\
\hline & & low fence & + & & & & & & & \\
\hline & Yuanzhe & high fence & + & $4110 \pm 912(25)$ & 0 & 0 & 0 & 2 & $5 \%$ & \\
\hline & & low fence & + & & & & & & & \\
\hline Non-fenced area & Kuaierma & - & - & $7223 \pm 546(10)$ & 0 & 0 & 0 & 0 & 0 & $51 \pm 17$ \\
\hline Significance & & & & * & & & & & & $*$ \\
\hline
\end{tabular}

a) +, Grassland fence had impacts on Przewalski's gazelle; -, no impacts on Przewalski's gazelles are found. Daily movement distance of the Przewalski's gazelle in fenced and non-fenced habitat differed significantly (Mann-Whitney Test, $U=5.27, d f=63, P=0.037$ ). Feeding bout duration of the Przewalski's gazelle in fenced and non-fenced habitat differed significantly (Mann-Whitney Test, $U=23.24, P<0.043$ ). * There was a significant difference between the fenced area group anr ther non-fenced area group. 
Table 3 Behavioral response of Przewalski's gazelle to grassland fence in different height $(\mathrm{Mean} \pm \mathrm{SE})^{\mathrm{a})}$

\begin{tabular}{lccc}
\hline \multirow{2}{*}{ High fence } & $\mathrm{JC}$ & $\mathrm{CT}$ & $\mathrm{WA}$ \\
\cline { 2 - 4 } & $0.00 \pm 0.00$ & $20.65 \pm 10.86$ & $49.61 \pm 27.27$ \\
\hline Low fence & $1.44 \pm 2.93$ & $0.07 \pm 0.26$ & $44.81 \pm 25.61$ \\
Percentage (\%) & 1.2 & 17.8 & 81.0 \\
Sample size & $n=168$ & $n=168$ & $n=168$ \\
Mann-Whitney $U$-test & $U=35.28^{*}$ & $U=45.00^{* *}$ & $U=20.40$ \\
Spearman correlation & $r=-0.80^{* *}$ & $r=-0.71^{* *}$ & $r=0.07, \mathrm{NS}$ \\
$P$ & 0.041 & 0.035 & 0.510 \\
\hline
\end{tabular}

a) * Difference (or Correlation) is significant at the 0.05 level (2-tailed); ** difference (or Correlation) is significant at the 0.01 level (2-tailed). JC, $\mathrm{CT}$ and WA: jumping across fences (JC), crawling through fences (CT) and walking along fences (WA), respectively. Spearman correlation was used to analyze the correlations between behavioral responses (JC, CT and WA) and height of grassland fence. The sample size we used in this correlation analysis was of 34 values that we measured in Hudong-Ketu and Yuanzhe respectively.

fenced area was much smaller than those live in unfenced area; secondly, grassland fence modified daily movement distance of Przewalski's gazelle; thirdly, height of grassland fence had clear impact on behavioral responses of the gazelle, and finally, grassland fence caused death directly and/ or indirectly in this gazelle.

Railroads, highways and pipelines cause habitat fragmentation and increase the risk of extinction of local populations in wild animals [1,2,26,27]. Previous studies have demonstrated that grassland fencing fragmented habitat and resulted in clear genetic variation in the Przewalski's gazelle [14,20,28]. Even in a designated nature reserve like the Chang Tang National Nature Reserve, grassland fencing is creating impasse and is causing casualty in wild ungulates [29]. The Hudong-Ketu population and the Yuanzhe population were originally a single population before the grassland fence was built in the early 1990s [14]. This population was not only isolated into two smaller sub-populations, but suffered adverse genetic effects [28]. Our field observations found that the longest distance moved of this gazelle in fenced habitat was about $5.0 \mathrm{~km}$ in Hudong-Ketu, however, the longest distance between the Hudong-Ketu and Yuanzhe was about $20 \mathrm{~km}$. We never found individuals that moved from Hudong-Ketu to Yuanzhe area during our field tracking surveys and our results show that habitat area of the local populations were clearly reduced after the grassland was fenced in the early 1990s (Figure 3). Recent reports on the genetic structure of the Przewalski's gazelle also indicated the anthropogenic landscape had deeper impacts on the population structure than lake and mountains [30,31].

Though studies had demonstrated that grassland fence lines did increase productivity of livestock and reduce the work of herding [9,32], such a measure may result in extirpation of wild ungulates from the fenced ranges. For example, movements of Antilocapra americana and Procapra gutturosa were affected by fences or railway lines [10,27]; grassland fencing affect feeding of sheep [33]. Our field observations also found that the longest distance moved of this gazelle in fenced habitat was about $5.0 \mathrm{~km}$ in $\mathrm{Hu}-$ dong-Ketu, however, the longest distance between the $\mathrm{Hu}-$ dong-Ketu and Yuanzhe was about $20 \mathrm{~km}$. We never found individuals that moved from Hudong-Ketu to Yuanzhe area during our field tracking surveys and our results show that habitat area of the local populations were clearly reduced after the grassland was fenced in the early 1990s (Figure 3). Shorter daily movement distance and the shrinking habitat area clearly indicate that the grassland fencing is endangering the survival of the gazelles in these two areas.

Behavioral responses of animals were used to evaluate whether they were affected by linear infrastructures [32,34]; and different characters of those linear features had varying impacts on wild animals $[32,33,35]$. Our results showed that Przewalski's gazelle responded to high and low grassland fencing differently. That is to say, the height of grassland fence was the key factor that influenced behaviors of Przewalski's gazelle. Gazelles respond in two ways when facing fence lines under $1.0 \mathrm{~m}$ high, however, if fence lines are higher than $1.0 \mathrm{~m}$, they prefer to walk along (WA) them, such behavioral response indicating that grassland fences under $1.0 \mathrm{~m}$ high had less impact on Przewalski's gazelles than those over $1.0 \mathrm{~m}$ high.

Death occurrence of Przewalski's gazelles in fenced habitat suggests that the height of fences had exceeded the maximum height which the gazelles can pass safely, and remaining near the livestock paddocks may facilitate predation by wolves. As impact of climate change is predominal on the Qinghai-Tibet Plateau, the gazelle trapped in the fenced grasslands are experiencing ever increasing danger of perishing [36].

\section{Conservation implications}

Large wild herbivores dwell on the steppes of Qinghai-Tibet Plateau are now diminished, mainly due to hunting activities; major social and economic changes have further accelerated the declining trend of large wild herbivores [37]. Our study revealed the devastating effects of grassland fencing on Przewalski's gazelle. Przewalski's gazelle is still threatened by habitat degradation and loss, habitat fragmentation, fencing, intensified competition with domestic livestock and predation. Further growth of this gazelle population is constrained by limited habitat availability and human-gazelle conflict [22]. We have also found the same devastating effects of grassland fence on Mongolian wild ass (Equus hemionus) and Tibetan gazelle (Procapra picticaudata) (unpublished data). Therefore, grassland wildlife management should be integrated into rangeland management schemes, particularly for those wild ungulates that need a 
large space to survive. Otherwise, we will almost certainly confront another wave of extinction of wild ungulates on the Central Asian grasslands.

We propose to develop integrated sustainable grassland management schemes in pastoral grassland ecosystems. Firstly, it is necessary to establish a nature reserve for those species, and remove all the grassland fence lines within the reserve. Secondly, we can replace wire grassland fence with soil grassland fence and decrease height of all grassland fence to $1.0 \mathrm{~m}$ for the target species to cross safely, and enable livestock to share the spatial resource with wild ungulates to maintain grassland biodiversity.

The authors thank Prof. Jia Zhiyun for his advices on revising this paper. This work was supported by the National Natural Science Foundation of China (31070469 and 31070348), the Key Program of Knowledge Innovation Program of Chinese Academy of Sciences (KSCX2-EW-Z-4), the Science and Technology Supporting Project of MOST (2008BAC39B04) and the Whitley Foundation for Nature.

1 Fahring L, Merriam G. Conservation of fragmented populations. Conser Biol, 1994, 8: 50-59

2 Saunder D A, Hobbs R J, Margules R. Biological consequences of ecosystem fragmentation, a review. Conserv Biol, 1991, 5: 18-32

3 Li C, Jiang Z, Feng Z, et al. Effects of highway traffic on diurnal activity of the critically endangered Przewalski's gazelle. Wildlife Res, 2009, 36: 379-385

4 Forman R T T, Alexander L E. Roads and their major ecological effects. Ann Rev Ecol Sys, 1998, 29: 207-231

5 Milner-Gulland E J, Lhagvasuren B. Population dynamics of the Mongolian gazelle Procapra gutturosa, an historical analysis. J Appl Ecol, 1998, 35: 240-251

6 State Development Planning Commission (SDPC). Report on the Population, Resources and Environment of China. Beijing: China Environmental Sciences Press, 1996. 74-77

7 State Environmental Protection Agency (SEPA). China Ecological and Environmental Situation Report 2002. Beijing: China Environmental Sciences Press, 2002. 12-14

8 Newman J L. Effects of woven wire fence with cattleguards on a free-living antelope population. Proc Antelope States Workshop, 1966, 2: 6-8

9 May M. Fencing for livestock management. Proc Biennial Antelope States Workshop, 1968. 3: 62-64

10 Gross B D, Holeches J L, Hallford D, et al. Effectiveness of antelope pass structures in restriction of livestock. J Range Manage, 1983, 36: 22-24

11 Dyer S J, O’Neill J P, Wasel S M, et al. Quantifying barrier effects of roads and seismic lines on movements of female woodland caribou in northeastern Alberta. Can J Zool, 2002, 80: 839-845

12 Poole D W, Western G, McKkillop I G. The effects of fence voltage and the type of conducting wire on the efficacy of an electric fence to exclude badgers (Meles meles). Crop Protection, 2004, 23: 27-33

13 Zhang R, Wang Z. Report on Mammals in Qinghai and Gansu Provinces. Beijing: Science Press, 1964

14 Jiang Z, Fen Z, Wang Z, et al. Historical and current distributions of Przewalski's gazelle. Acta Theriol Sin, 1995, 15: 241-245

15 Jiang Z, Li D, Wang W. Population declines of Przewalski’s gazelle around Qinghai Lake, China. Oryx, 2000, 34: 129-135

$16 \mathrm{Hu}$ J, Jiang Z. Predicting the potential distribution of the endangered Przewalski's gazelle. J Zool, 2010, 28: 54-63

17 Li Z, Jiang Z. Grouping behavioral differences in the sympatric Tibetan gazelle and Przewalski's gazelle in the upper Buha River region. Zool Res, 2006, 27: 396-402

18 Jiang Z, Li D, Peng J, et al. Structure, elasticity and diversity of animal behaviour. Biodiversity Sci, 2001, 9: 265-274

19 Liu B, Jiang Z. Diet composition of wolf in the Qinghai Lake region in northeast Tibetan Plateau. Acta Theriol, 2002, 48: 255-263

20 Li D, Jiang Z, Wang W. Impacts of human activities on the distribution of the critically endangered Przewalski's gazelle. Acta Ecol Sin, 1999, 19: 890-895

21 You Z, Jiang Z. Courtship and mating behaviors in Przewalski's gazelle (Procapra przewalskii). Acta Zool Sin, 2005, 51: 187-194

22 Li C, Jiang Z, Ping X, et al. Current status and conservation of Przewalski's gazelle Procapra przewalskii. Oryx, 2012, 46: 145-153

23 You Z, Jiang Z, Li C. Location of rut stands vs. mating opportunities in Przewalski's gazelle: A field test of the "Resource-based hypothesis" and "Female traffic version of the hotspot hypothesis". Curr Zool, 2011, 57: 701-708

24 Prejevalsky [Przewalski] N. Mongolia, the Tangut Country, and the Solitudes of Northern Tibet. London, Sampson, Low, Marston, Searle, and Rivington, 1876

25 Li D, Jiang Z. Population viability analysis for the Przewalski's gazelle (Procapra przewalskii). Russian J Ecol, 2001, 33: 115-120

26 Schroeder M A, Robb L A. Fidelity of greater sage-grouse Centrocercus urophasianus to breeding areas in a fragmented landscape. Wildlife Biol, 2003, 9: 291-299

27 Takehiko Y I, Naoko M, Badamjav L, et al. Preliminary evidence of a barrier effect of a railroad on the migration of Mongolian gazelles. Conserv Biol, 2005, 19: 945-948

28 Lei R, Jiang Z. Phylogeography and genetic diversity of the critically endangered Przewalski's gazelle. Anim Conserv, 2003, 6: 361-367

29 Fox J L, Kelsang D, Dorji T. Tibetan antelope Pantholops hodgsonii conservation and new rangeland management policies in the western Chang Tang Nature Reserve, Tibet: Is fencing creating an impasse? Oryx, 2009, 43: 183-190

30 Yang J, Jiang Z. Genetic diversity, population genetic structure and demographic history of Przewalski's gazelle (Procapra przewalskii): Implications for conservation. Conserv Genetics, 2011, doi: 10.1007/ s10592-011-0244-7

31 Yang J, Jiang Z, Zeng Y, et al. Effect of anthropogenic landscape features on population genetic differentiation of Przewalski's gazelle: Main role of human settlement. PLoS One, 2011, 6: e20144

32 Goddard P J, Summers R W, Macdonald A J. Behavioral responses of red deer to fences of five different designs. Appl Anim Behav Sci, 2001, 73: 289-298

33 Wang S, Li Y, Wang Y. Relationship between foraging areas of sheep (wether) and spatial heterogeneity of grassland landscape. Acta Ecol Sin, 1999, 19: 25-30

34 Hodgetts B V, Waas J R, Matthews L R. The effects of visual and auditory disturbance no the behavior of red deer (Cervus elaphus) at pasture with and without shelter. Appl Anim Behav Sci, 1998, 55: 337-351

35 Baines D, Andrew M. Marking of deer fences to reduce frequency of collisions by woodland grouse. Conserv Biol, 2003, 110: 169-176

$36 \mathrm{Hu}$ J, Jiang Z. Climate change hastens the conservation urgency of an endangered ungulate. PLoS One, 2011, 6: e22873

37 Mallon D P, Jiang Z. Grazers on the plains: Challenges and prospects for large herbivores in Central Asia. J Appl Ecol, 2009, 46: 516519

Open Access This article is distributed under the terms of the Creative Commons Attribution License which permits any use, distribution, and reproduction in any medium, provided the original author(s) and source are credited. 